\title{
Microstructural Impact of Si and Ni During High Temperature Quenching and Partitioning Process in Medium-Mn Steels
}

\author{
S. AYENAMPUDI, C. CELADA-CASERO, Z. ARECHABALETA, M. ARRIBAS, \\ A. ARLAZAROV, J. SIETSMA, and M.J. SANTOFIMIA
}

\begin{abstract}
Austenite stabilization through carbon partitioning from martensite into austenite is an essential aspect of the quenching and partitioning (Q\&P) process. Substitutional alloying elements are often included in the chemical composition of Q\&P steels to further control the microstructure development by inhibiting carbide precipitation (silicon) and further stabilize austenite (manganese and nickel). However, these elements can interfere in the microstructure development, especially when high partitioning temperatures are considered. In this study, the microstructural development during the Q\&P process of four low-carbon, medium-manganese steels with varying contents of silicon and nickel is investigated. During partitioning at $400{ }^{\circ} \mathrm{C}$, silicon hinders cementite precipitation in primary martensite thereby assisting carbon partitioning from martensite to austenite. During partitioning at temperatures of $500{ }^{\circ} \mathrm{C}$ and $600{ }^{\circ} \mathrm{C}$, presence of nickel inhibits pearlite formation and promotes austenite reversion, respectively. It is observed that the stabilization of austenite is significantly enhanced through the addition of nickel by slowing down the kinetics of competitive reactions that are stimulated during the partitioning stage. Results of this study provide an understanding of the interplay among carbon, silicon and nickel during Q\&P processing that will allow the development of new design strategies to tailor the microstructure of this family of alloys.
\end{abstract}

https://doi.org/10.1007/s11661-021-06144-5

(C) The Author(s) 2021

\section{INTRODUCTION}

STEEL grades with high strength and ductility are a paramount requirement for the automotive industry to reduce the consumption of fuel and enhance passenger safety. ${ }^{[1]}$ Consequently, for the past few years, special interest has been directed towards the research and development of novel advanced high-strength steels (AHSS) such as quenching and partitioning (Q\&P) steels, which have a good combination of high strength and ductility. Speer et al. ${ }^{[2]}$ introduced the Q\&P process in which an austenitic microstructure (partial or complete) is quenched to a temperature below the

S. AYENAMPUDI, C. CELADA-CASERO, J. SIETSMA, M.J. SANTOFIMIA are with the Department of Materials Science and Engineering, Delft University of Technology, Mekelweg 2, 2628 CD, Delft, The Netherlands. Contact e-mail: S.Ayenampudi@tudelft.nl Z. ARECHABALETA and M. ARRIBAS are with the Basque Research and Technology Alliance (BRTA), Astondo Bidea, Edificio 700, 48160 Derio, Bizkaia, Spain. A. ARLAZAROV is with the ArcelorMittal Maziûres Research, Voie Romaine, Maiziû́res-lû́s-Metz, 57283, France.

Manuscript submitted October 2, 2020, accepted December 20, 2020.

Article published online February 17, 2021 martensitic start temperature $\left(M_{\mathrm{s}}\right)$ to form a microstructure with a certain fraction of austenite $(\gamma)$ and primary martensite (M1). The process is followed by heating up the steel to a temperature above the initial quenching temperature $\left(T_{\mathrm{Q}}\right)$ and isothermal holding for a certain time. This is termed the partitioning stage during which carbon partitions from supersaturated martensite to untransformed austenite. During the final quench, the fraction of austenite that is deficient in carbon will transform into fresh martensite (M2).

In advanced high-strength steels, the retained austenite present in the microstructure is considered a key phase that determines the ductility of the material. ${ }^{[3-5]}$ Therefore, most of the earlier studies regarding the Q\&P process were focused on retaining high fractions of retained austenite by either the addition of austenite stabilizing alloying elements or tailoring the partitioning conditions. Austenite stabilization via carbon, an interstitial alloying element, has been studied extensively over the past few years. ${ }^{[2,5-11]}$ Some recent studies ${ }^{[12-18]}$ reported austenite stabilizing effects of substitutional alloying elements such as manganese and nickel on the final Q\&P microstructures. In a recent study by Kim et al. ${ }^{[17]}$ three medium-carbon low-manganese steels with different nickel contents (0 to 2 wt pct) were 
studied. It was reported that, with an increase in nickel content, the volume fraction and stability of retained austenite were enhanced. An enhancement in mechanical properties with increasing partitioning temperature was also reported. Rizzo et al. ${ }^{[18]}$ studied four different steels with different carbon and nickel contents after partitioning at $400{ }^{\circ} \mathrm{C}$ for times up to $300 \mathrm{~s}$. The resulting Q\&P microstructures showed an increase in retained austenite fractions, and an improvement in strength and ductility was reported with an increase in nickel content. These investigations ${ }^{[12-18]}$ show that the composition of the steel, majorly substitutional alloying elements, plays a crucial role in the control of the microstructure and mechanical properties of Q\&P steels.

Recently, Pierce et al. ${ }^{[19]}$ studied two $0.2 \mathrm{C}-1.5 \mathrm{Mn}-1.3 \mathrm{Si}$ steels with $1.5 \mathrm{wt}$ pet chromium and nickel, respectively. It was observed that at short isothermal holding times, with an increase in partitioning temperature from $350{ }^{\circ} \mathrm{C}$ to $500{ }^{\circ} \mathrm{C}$, an increase in the fraction of retained austenite occurred. Moreover, it was also observed that a higher fraction austenite was stabilized in the alloy with chromium compared to the alloy with nickel. Ding et al. ${ }^{[20]}$ showed that partitioning at temperatures $>\mathrm{Ac}_{1}\left(660{ }^{\circ} \mathrm{C}\right)$ can lead to austenite reverse transformation promoting manganese partitioning into the newly formed austenite. The above studies emphasize the advantages of employing high-temperature partitioning cycles and the quantitative impact of substitutional alloying elements on retaining austenite in the final Q\&P microstructures. However, the interactions of these alloying elements with respect to austenite stabilization and their effects on the microstructure development during the Q\&P process were seldom addressed in the previous works.

The present work focuses on understanding the impact of the substitutional alloying elements nickel and silicon on the microstructural development of four low-carbon medium-manganese steels during the application of $\mathrm{Q} \& \mathrm{P}$ treatments. Partitioning temperatures of $400{ }^{\circ} \mathrm{C}, 500{ }^{\circ} \mathrm{C}$ and $600{ }^{\circ} \mathrm{C}$ in a time range of 60 to 3600 seconds are considered. The insights gained from this study provide an enhanced understanding of the mechanisms involved during the high-temperature Q\&P process and help in developing new physical and chemical design strategies for Q\&P steels.

\section{EXPERIMENTAL PROCEDURE}

\section{A. Materials and Methods}

Four low-carbon medium-manganese steels with varying nickel and silicon contents were investigated. Manganese and nickel were added to the composition to delay the phase transformations, like ferrite and bainite, during the partitioning stage, while silicon was added to suppress carbide formation. Moreover, the addition of substitutional alloying elements such as manganese and nickel aid in increasing the thermal stability of austenite facilitating high-temperature Q\&P cycles. The compositions are listed in Table I and are named $M n, M n S i$, $M n N i$ and $M n S i N i$.
Initially, steel ingots weighing $15 \mathrm{~kg}$ were produced using a vacuum induction melting furnace. The ingots were reheated to $\sim 1250{ }^{\circ} \mathrm{C}$ and subsequently hot-rolled down to a final thickness of $4 \mathrm{~mm}$.

Specimens with $10 \times 4 \times 4 \mathrm{~mm}^{3}$ dimensions were machined with the longer dimension parallel to the rolling direction and used for performing Q\&P heat treatments in a Bähr 805 DIL A/D dilatometer. The sample temperature was controlled and monitored using a type $\mathrm{S}$ thermocouple that was spot-welded on the surface. During the heating and isothermal segments, a vacuum of approximately $10^{-4}$ mbar was induced in the sample chamber while helium was used as the coolant during the cooling segments.

For microstructural investigations on the heat-treated samples, a JEOL JSM-6500F field emission gun scanning electron microscope (FEG-SEM) was used with an accelerating voltage of $15 \mathrm{kV}$. FEG-SEM specimens were prepared by following standard grinding, polishing and etching with Nital 2 pct.

A Bruker type D8-Advance diffractometer was used to calculate the volume fraction $\left(f_{\mathrm{RA}}\right)$ and lattice parameter $\left(a_{\gamma}\right)$ of retained austenite at room temperature. The diffraction angles covered during the measurements are in the range of $40 \mathrm{deg}<2 \theta<130 \mathrm{deg}$, with Co $K \alpha$ radiation, where the (110) $\alpha,(200) \alpha$, (211) $\alpha$, $(220) \alpha$ and the $(111) \gamma,(200) \gamma,(220) \gamma,(311) \gamma$ peaks are covered within this $2 \theta$ range. A $0.042 \mathrm{deg} 2 \theta$ step size with a counting per step of 3 seconds was used. Following the direct comparison method of austenite and martensite peaks suggested by Jatczak, ${ }^{[21]}$ the retained austenite volume fractions and the corresponding uncertainties were calculated. To determine the carbon concentration of retained austenite from its lattice parameter, the equations proposed by Dyson and Holmes $^{[22]}$ and van Dijk et al. ${ }^{[23]}$ were combined and adjusted to suit the steel compositions in the current work:

$$
\begin{aligned}
a_{\gamma}= & 3.556+0.0453 \cdot x_{\mathrm{C}}+0.00095 \cdot x_{\mathrm{Mn}}+0.00157 \cdot x_{\mathrm{Si}} \\
& +0.0006 \cdot x_{\mathrm{Cr}}-0.0002 \cdot x_{\mathrm{Ni}}
\end{aligned}
$$

where $x_{i}$ represents the concentration of the alloying element $i$ in wt pct and the lattice parameter is in units of $\AA$. If any partitioning of substitutional alloying elements from martensite into austenite occurred, it would have been very local, and its influence on the overall lattice parameter and thus calculation of the average chemical composition of austenite would have been negligible. Hence, the nominal composition of the alloy and lattice parameter of retained austenite was used to calculate the carbon content of retained austenite.

A 7307 vibrating sample magnetometer was used to perform room temperature magnetic measurements. A cubic specimen, cut from the heat-treated dilatometry sample with an edge dimension of $2.0 \mathrm{~mm}$, was used for the measurements. For calibration purposes, a standard NIST (National Institute of Standards and Technology) nickel specimen was used. Room temperature magnetic measurements were performed by varying the applied magnetic field from +1.6 to $-1.6 \mathrm{~T}$ at an interval of 
$0.15 \mathrm{~T}$. According to the method described by Zhao et al. ${ }^{[24]}$ the total volume fraction of non-FCC phases in the quenched specimen was determined by comparing the values of saturation magnetization of both the pure $\mathrm{Fe}-\mathrm{BCC}$ specimen, which was $215 \mathrm{Am}^{2} / \mathrm{kg}$ at room temperature ${ }^{[25]}$ and the specimen with martensite that was to be measured. The error in estimating the volume fraction from magnetic measurements was estimated as \pm 0.01 .

\section{B. Design of Heat Treatments}

To identify the Q\&P process parameters, such as the austenitization and quenching temperatures $\left(T_{\gamma}, T_{Q}\right)$, the $\mathrm{Ac}_{1}$ and $\mathrm{Ac}_{3}$ temperatures were characterized. A specimen of each alloy was subjected to full austenitization by heating to a temperature of $950{ }^{\circ} \mathrm{C}$ at $10{ }^{\circ} \mathrm{C} / \mathrm{s}$. After 120 seconds of isothermal holding, the sample was directly quenched to room temperature at a cooling rate of $30^{\circ} \mathrm{C} / \mathrm{s}$. As explained in the work of Reference 26 the $\mathrm{Ac}_{1}$ and $\mathrm{Ac}_{3}$ temperatures of the four alloys were determined from the first derivative of the dilatometry curve with respect to time. After the identification of $\mathrm{Ac}_{1}$ and $\mathrm{Ac}_{3}$ temperatures, a new set of heat treatments on all alloys with austenitization temperatures of $\mathrm{Ac}_{3}+$ $50{ }^{\circ} \mathrm{C}$ was performed to identify the martensite start temperature and the martensite formation kinetics during quenching to room temperature. Table II gives information about the transformation temperatures of all four alloys.

Figure 1(a) shows the relative change in length with temperature during the cooling stage of the as-quenched specimens of the four alloys. A quenching followed by reheating heat treatment was performed to characterize the expansion behavior of the quenched microstructure of the alloys. The change in length behavior during the reheating cycle gives information on the thermal expansion of the as-quenched specimen consisting of a mixture of BCC and FCC phases. As shown in Figure 1(a), the volume fraction of martensite at room temperature and

Table I. Chemical Composition (Wt Pet) of Q\&P Steels Investigated in the Current Work

\begin{tabular}{lccccc}
\hline Alloy & $\mathrm{C}$ & $\mathrm{Mn}$ & $\mathrm{Si}$ & $\mathrm{Ni}$ & $\mathrm{Fe}$ \\
\hline $\mathrm{Mn}$ & 0.19 & 6.0 & - & - & bal. \\
$\mathrm{MnSi}$ & 0.19 & 5.8 & 1.4 & - & bal. \\
$\mathrm{MnNi}$ & 0.19 & 6.0 & - & 2.1 & bal. \\
$\mathrm{MnSiNi}$ & 0.19 & 5.7 & 1.4 & 1.6 & bal. \\
\hline
\end{tabular}

its evolution with temperature (Figure 1(b)) was obtained considering the linear expansion of the FCC and $\mathrm{BCC}$ lattice and applying the lever rule on the respective dilatometry curves. According to the room temperature magnetometer measurements, the as-quenched samples of $\mathrm{Mn}, \mathrm{MnSi}, \mathrm{MnNi}$ and $\mathrm{MnSiNi}$ alloys comprise about $0.94,0.93,0.92$ and $0.93( \pm 0.01)$ fractions of martensite, respectively. These martensite fractions correspond to the final change in length observed at room temperature, as indicated for $\mathrm{MnNi}$ alloys in Figure 1(a). Magnetic measurements are performed to calculate the austenite fraction in the as-quenched specimen where an in-bulk estimation of the total volume fraction of untransformed austenite is required to characterize the martensite transformation kinetics during the first quench. Based on the data from Figure 1(b), formation of a 0.75 volume fraction of martensite is selected as the quenching temperature $\left(T_{\mathrm{Q}}\right)$ in all the Q\&P heat treatments performed in the current work.

The four steels were then Q\&P processed as indicated in Figure 2. The specimens were heated to a temperature of $\mathrm{Ac}_{3}+50{ }^{\circ} \mathrm{C}$ and cooled down to a quenching temperature $\left(T_{Q}\right)$ after 120 seconds of isothermal holding. This lead to formation of austenite/martensite phases in the ratio of $0.25 / 0.75$. The theoretical optimum quenching temperature, calculated using the $\mathrm{CCE}$ model, ${ }^{2]}$ for the four alloys corresponds to 0.70 to 0.80 volume fraction of primary martensite. However, for the comparison of different alloys, it is important to have a similar volume fraction of primary martensite/austenite at the beginning of the partitioning stage. Hence, 0.75 volume fraction of primary martensite is selected. The initial quenching temperatures $\left(T_{Q}\right)$ are different for all four steel compositions and are shown in Figure 1(b). Thereafter, the specimens were subjected to a partitioning stage, where partitioning temperatures $\left(T_{\mathrm{p}}\right)$ of $400{ }^{\circ} \mathrm{C}, 500{ }^{\circ} \mathrm{C}$ and $600{ }^{\circ} \mathrm{C}$ were applied for partitioning times $\left(t_{\mathrm{p}}\right)$ up to 3600,900 and 300 seconds, respectively. The partitioning temperatures range from typical values aiming only for the austenite stabilization through carbon partitioning $\left(400{ }^{\circ} \mathrm{C}\right)$ to a temperature of $600{ }^{\circ} \mathrm{C}$, which can promote austenite stabilization through partitioning of substitutional alloying elements. The highest partitioning temperature $\left(600^{\circ} \mathrm{C}\right)$ applied in the Q\&P heat treatments of the current work is below the measured $\mathrm{Ac}_{1}$ for all four alloys (Table II). In their earlier work, the same authors ${ }^{[27]}$ studied the microstructure development in a Fe-0.31C-4.5Mn-1.5Si steel during Q\&P processing in which the partitioning

Table II. Phase Transformation Temperatures of All Four Alloys Investigated in the Current Work

\begin{tabular}{lcccc}
\hline Alloy & $\mathrm{Ac}_{1}\left( \pm 5^{\circ} \mathrm{C}\right)$ & $\mathrm{Ac}_{3}\left( \pm 5^{\circ} \mathrm{C}\right)$ & $\mathrm{M}_{\mathrm{s}}\left( \pm 5^{\circ} \mathrm{C}\right)$ & $\mathrm{T}_{\mathrm{Q}}\left( \pm 5^{\circ} \mathrm{C}\right)$ \\
\hline Mn & 700 & 780 & 285 & 150 \\
MnSi & 716 & 812 & 260 & 140 \\
MnNi & 680 & 745 & 262 & 110 \\
MnSiNi & 710 & 790 & 266 & 120 \\
\hline
\end{tabular}




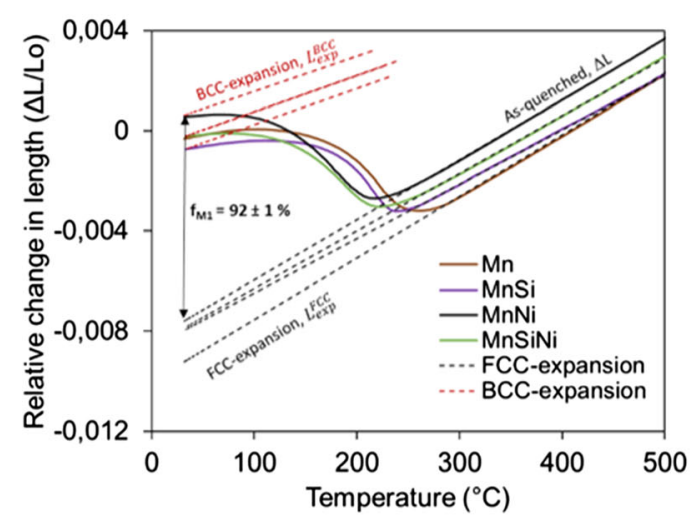

(a)

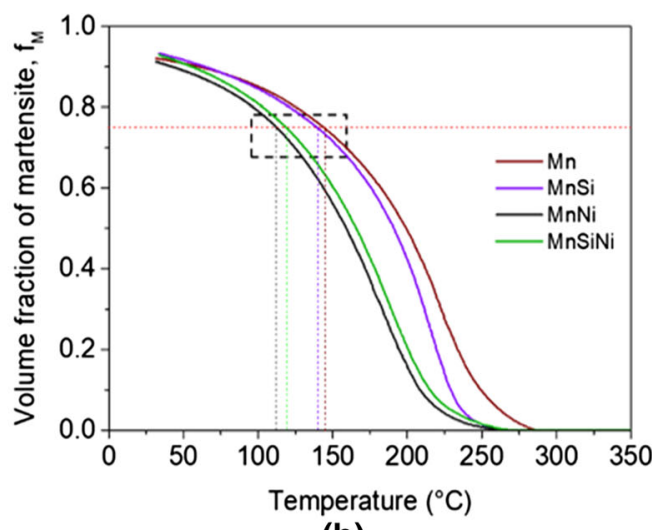

(b)

Fig. 1-(a) Change in length versus temperature curve during quenching for $\mathrm{Mn}, \mathrm{MnSi}, \mathrm{MnNi}$ and $\mathrm{MnSiNi}$ alloys. In the same figure the fraction of martensite $\left(f_{\mathrm{M}}\right)$ formed in $M n N i$ alloy at the end of as-quenched heat treatment is shown. $(b)$ Volume fraction of martensite as a function of temperature from the as-quenched dilatation curve for $\mathrm{Mn}, \mathrm{MnSi}, \mathrm{MnNi}$ and $\mathrm{MnSiNi}$ alloys.

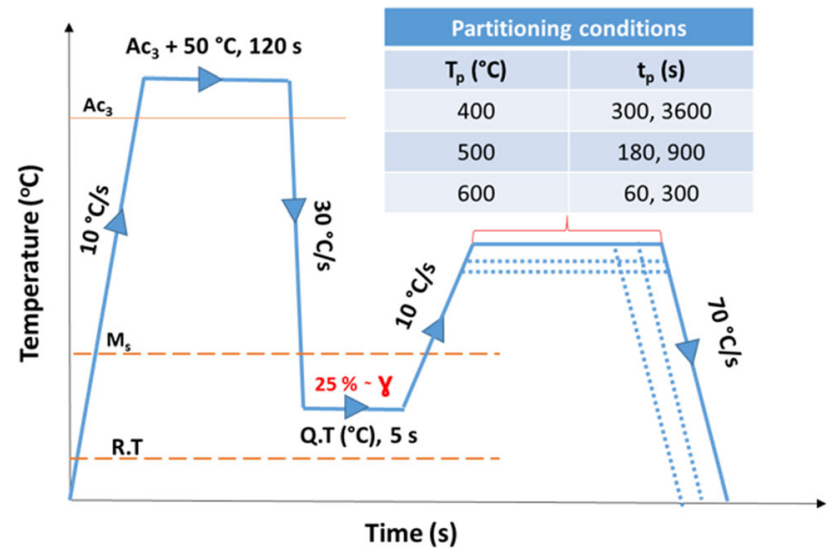

Fig. 2-Schematic representation of Q\&P thermal cycles applied on $\mathrm{Mn}, \mathrm{MnSi}, \mathrm{MnNi}$ and $\mathrm{MnSiNi}$ alloys in the present work.

temperatures and times were in a range of $400{ }^{\circ} \mathrm{C}$ to 600 ${ }^{\circ} \mathrm{C}$ and 60 to 3600 seconds, respectively. It was observed that, during the partitioning at higher temperatures (500 ${ }^{\circ} \mathrm{C}$ to $600{ }^{\circ} \mathrm{C}$ ), minimization of competitive reactions, such as precipitation of carbides in austenite and formation of pearlite, is effective when the partitioning times are kept to $<300$ seconds. Hence, in the current work, shorter partitioning times were used at higher partitioning temperatures. As a last step in the heat treatment, the specimens were cooled down to room temperature at a cooling rate of $70{ }^{\circ} \mathrm{C} / \mathrm{s}$. In the following sections the specimens will be referred to as QP $T_{\mathrm{P}}-t_{\mathrm{P}}$ for easy interpretation of the heat treatments.

\section{RESULTS}

\section{A. Phase Fractions and Carbon Content of Retained Austenite}

Figure 3 shows the dilatation curve of the as-quenched specimen (black dashed line) and quenching and partitioning cycle of the QP400-3600 condition (solid red line) of $M n N i$ alloy. The black solid lines

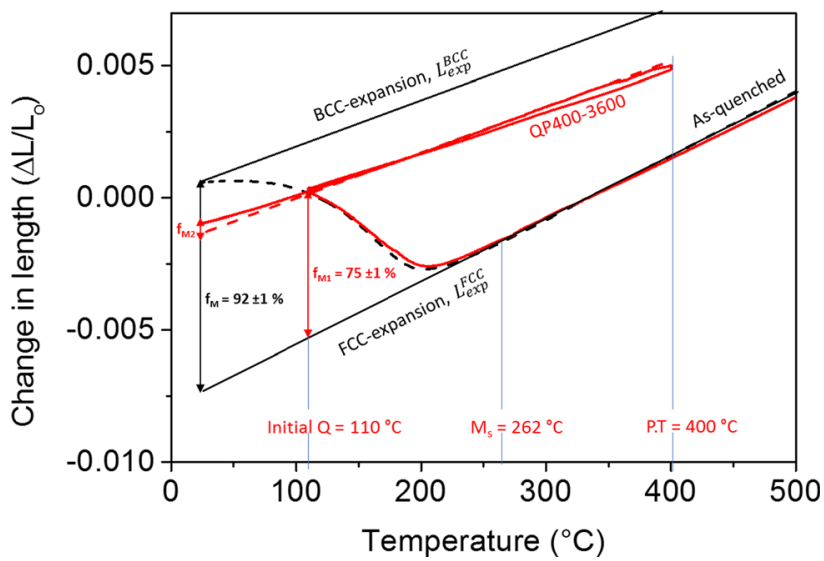

Fig. 3-Representation of relative change in length vs temperature for an as-quenched heat treatment (dashed line) and QP400-3600 heat treatment (solid line) of $M n N i$ alloy. $f_{\mathrm{M}}$ is the fraction of martensite formed after an as-quenched heat treatment; $f_{\mathrm{M} 1}$ and $f_{\mathrm{M} 2}$ are the fractions of primary and fresh martensite formed during QP400-3600 heat treatment, respectively.

indicate the BCC and FCC linear expansion lines obtained from the quenching + reheating heat treatment. The red dashed line indicates the BCC linear expansion line that is shifted to match the final quenching to calculate the volume fraction of fresh martensite. This figure is used to explain the manner in which the microstructural development is studied by dilatometry during Q\&P processing routes. Evaluation of the QP400-3600 dilatometry curve in Figure 3 shows that the only phases forming during cooling from austenitization are primary martensite $\left(f_{\mathrm{M} 1}\right)$ during the initial quench and fresh martensite $\left(f_{\mathrm{M} 2}\right)$ during the final quench. The phenomena that occurred during the partitioning stage are discussed in detail in the following sections.

In all the Q\&P heat treatments, the volume fraction of primary martensite $\left(f_{\mathrm{M} 1}\right)$ is kept the same, i.e., $0.75 \pm$ 0.01. Comparison of change in length measured during the final quench with that of the directly quenched specimen allows us to calculate the volume fraction of 
fresh martensite $\left(f_{\mathrm{M} 2}\right)$. Since fresh martensite formation occurs from a carbon-enriched austenite, an average error of up to \pm 0.01 in the evaluation of volume fraction of fresh martensite is estimated based on Reference 28. The X-ray diffractometer technique was used to measure the volume fraction of retained austenite $\left(f_{\mathrm{RA}}\right)$ in the final microstructures. X-ray diffraction measurements are performed in the cases where the lattice parameter of retained austenite is required to calculate the carbon content in the retained austenite. The volume fraction of the rest of the final microstructural constituents, such as pearlite $\left(f_{\mathrm{p}}\right)$, was calculated by balancing the phase fractions:

$$
f_{\mathrm{M} 1}+f_{\mathrm{M} 2}+f_{\mathrm{RA}}+f_{\mathrm{p}}=1
$$

The application of the above-described method led to the results displayed in Figure 4, which shows the volume fraction of phases in the final Q\&P microstructures of all four alloys. In some cases, the total volume fraction of retained austenite and fresh martensite in the final microstructure has a deviation of up to \pm 0.01 . This deviation is observed to be within the error of measurements and calculations. Hence, the effect is considered negligible.

Figure 4(a) shows the volume fractions of retained austenite (RA), fresh martensite (M2) and pearlite (P) in the final microstructures and carbon contents in the

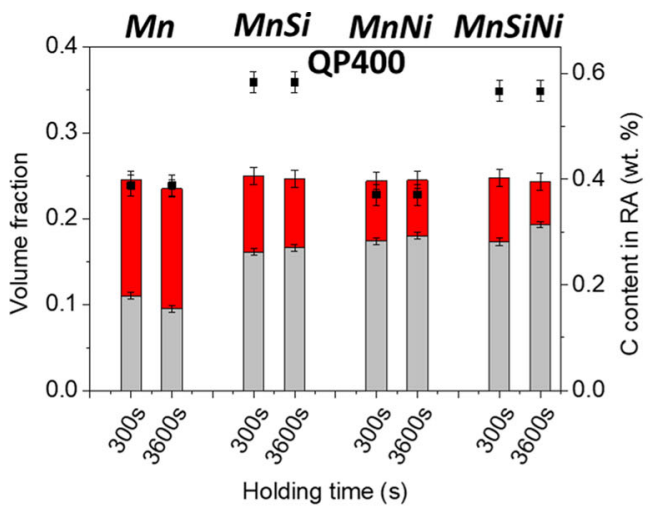

(a)

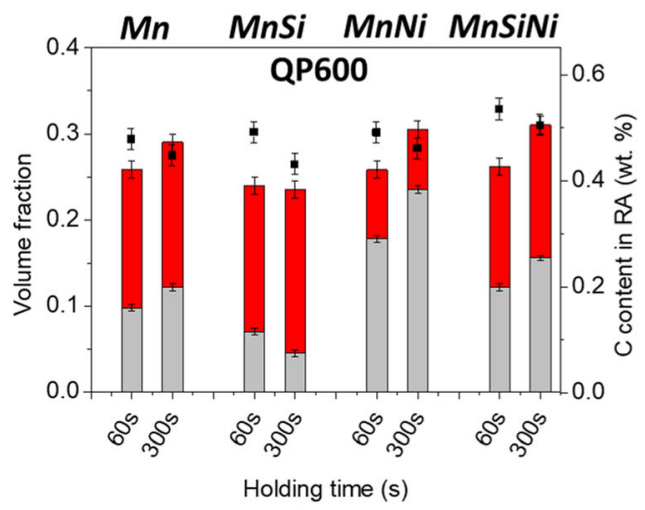

(c) retained austenite of all four alloys at the end of QP400-300 and QP400-3600 seconds heat treatments. A slight increase in the volume fraction of retained austenite with partitioning time is detected in all the alloys except for $\mathrm{Mn}$ alloy. Alloys with nickel $(\mathrm{MnNi}$ and $\mathrm{MnSiNi}$ ) show the lowest fractions of fresh martensite and highest fractions of retained austenite. Alloys with silicon show higher carbon content (wt pet) in retained austenite compared to that of alloys without silicon.

Figure 4(b) displays the volume fractions of the phases in the final Q\&P microstructure of the four alloys after partitioning at $500{ }^{\circ} \mathrm{C}$ for 180 and 900 seconds. With an increase in partitioning time, the volume fraction of fresh martensite increases. After 900 seconds of isothermal holding an almost similar volume fraction of pearlite is observed in $\mathrm{Mn}, \mathrm{MnSi}$ and $\mathrm{MnSiNi}$ alloys. With the increase in the volume fraction of pearlite, the carbon content and volume fraction of retained austenite decrease. The lowest and highest fraction of retained austenite is respectively detected in $M n$ alloy after partitioning for 900 seconds and by $\mathrm{MnNi}$ alloy after partitioning for 180 seconds.

Figure 4(c) shows the volume fractions of the final microstructural constituents of all alloys after partitioning at $600{ }^{\circ} \mathrm{C}$ for 60 and 300 seconds. Except for $\mathrm{MnSi}$ alloy, a clear increase of the volume fraction of retained austenite with the partitioning time is observed.

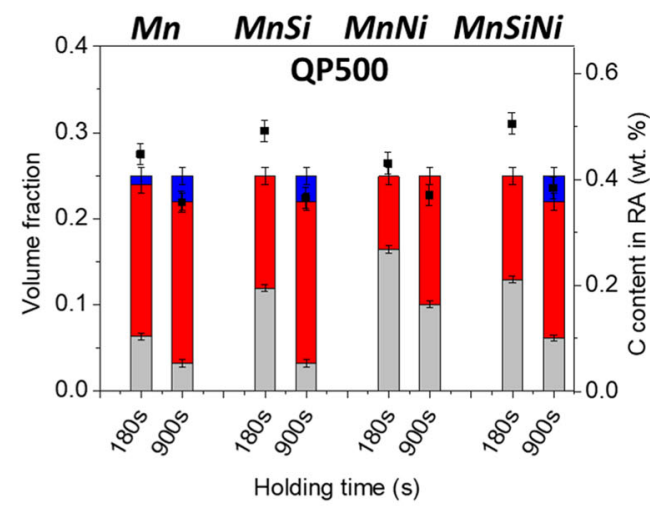

(b)

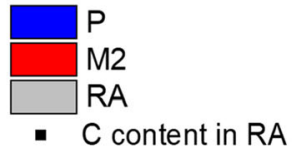

Fig. 4-Representation of the volume fraction of phases present at the end of (a) QP400, (b) QP500 and (c) QP600 heat treatments as a function of the partitioning time for $\mathrm{Mn}, \mathrm{MnSi}, \mathrm{MnNi}$ and $\mathrm{MnSiNi}$ alloys. Carbon content of retained austenite after respective Q\&P heat treatments is also represented in the same figure. P: pearlite; M2: fresh martensite; RA: retained austenite. 
Interestingly, after 300 seconds of partitioning time at $600{ }^{\circ} \mathrm{C}$, a volume fraction of retained austenite of around 0.24 was measured in the $M n N i$ alloy. The total volume fraction of retained austenite, fresh martensite and pearlite exceeds the initial volume fraction of austenite at the beginning of the partitioning stage, which was 0.25 . This indicates a reduction in the volume fraction of primary martensite during the partitioning step, which could be due to an austenite reverse transformation (ART). In all the alloys, a decrease in carbon content (wt pct) in retained austenite is observed with partitioning time.

\section{B. Microstructural Characterization}

The final microstructures of $\mathrm{Mn}, \mathrm{MnSi}, \mathrm{MnNi}$ and $M n S i N i$ alloys after QP400-3600 heat treatment are shown in Figures 5(a) through (d). Retained austenite films and fresh martensite/retained austenite islands (MA islands) are observed as the common microstructural features in all four alloys. In alloys without silicon ( $M n$ and $M n N i$ alloys), a higher degree of carbide precipitation within primary martensite is observed. In addition, in $M n$ alloy, precipitation of carbides along the grain boundaries of prior austenite is observed. In alloys with silicon ( $\mathrm{MnSi}$ and $\mathrm{MnSiNi}$ ), primary martensite islands devoid of carbides are observed.

SEM micrographs (Figures 5(e) to (h)) of all the four alloys after partitioning at $500{ }^{\circ} \mathrm{C}$ for 900 seconds show the presence of MA islands and primary martensite with carbides as a common feature. The SEM micrograph of $M n$ alloy confirms the presence of pearlite along the grain boundaries of prior austenite/martensite, which is also observed in $M n S i$ and $M n S i N i$ alloys. A parallel array of carbides (indicated by dashed lines) aligned in certain directions is observed as a common microstructural feature in alloys with silicon ( $\mathrm{MnSi}$ and $\mathrm{MnSiNi}$ ). These arrays of precipitates are located in between primary martensite laths, which suggests that they form inside austenite films. In $M n N i$ alloy, primary martensite is clearly enriched with carbides while retained austenite films present in between the martensite laths are observed.

The SEM micrographs of $M n, M n N i$ and $M n S i N i$ alloys (Figures 5(i), (k) and (1), respectively) after QP600-300 heat treatments show significant retained austenite film distribution in between the martensite laths. Moreover, retained austenite islands are observed in $M n N i$ alloy. In $M n S i N i$ alloy, carbide precipitation along grain boundaries of prior austenite is observed.

Interestingly, the SEM micrograph of $M n S i$ alloy does not display evidence of a significant amount of carbides inside the primary martensite. However, precipitation of carbides inside austenite films is observed. Also, the micrograph of $M n S i$ alloy suggests that prior austenite grains are much smaller than in the rest of the alloys. Silicon increases the $\mathrm{Ac}_{3}$ temperature but barely affects the $\mathrm{Ac}_{1}$ temperature, and the austenitization range is much wider in alloys with silicon. ${ }^{[29]}$ Hence, $\mathrm{Ac}_{3}+50{ }^{\circ} \mathrm{C}$ treatment leads to a microstructure with a smaller prior austenite grain size in $M n S i$ alloy compared to the other alloys. Celada-Casero et al. ${ }^{[30]}$ showed that the refined microstructure after the initial quench enhances the partitioning of carbon into austenite from martensite during the partitioning stage. This can also explain the reason for the sparse distribution of carbides in primary martensite in alloys with silicon.

\section{Dilatometry}

During the partitioning stage of QP400-3600 heat treatment (Figure 6(a)), an expansion in the change in length for the initial 100 seconds of partitioning is observed, which is an effect of carbon partitioning from primary martensite (M1) into austenite. ${ }^{[31]}$ This expansion is followed by a contraction in the case of alloys without silicon ( $M n$ and $M n N i$ ), which is attributed to carbide precipitation in primary martensite. ${ }^{[17]}$ In the alloys with silicon ( $\mathrm{MnSi}$ and $\mathrm{MnSiNi}$ ) expansion is followed by a plateau indicating almost no carbide precipitation, which is consistent with SEM observations (Figures 5(b) and (d)).

Figure 6(b) shows the change in length with time detected during partitioning at $500{ }^{\circ} \mathrm{C}$ for 900 seconds. The dilatometry curves of alloys without silicon ( $\mathrm{Mn}$ and $M n N i$ ) show a small expansion for the first 50 seconds, indicating carbon partitioning. In the later part of the curve, $M n$ alloy shows a slight expansion, whereas $\mathrm{MnNi}$ alloy displays a slight contraction. Observations from the SEM micrographs (Figures 5(e) and (g)) suggest that the gradual expansion is related to pearlite formation and a slight contraction is related to carbide precipitation in primary martensite. Both alloys with silicon ( $M n S i$ and $M n S i N i$ ) exhibit a similar decrease in change in length followed by a plateau. Looking at the SEM micrographs (Figures 5(f) and (h)), it is considered that this decrease could be a reaction to the formation of a fine and parallel array of carbides. Based on experimental observations and the theoretical calculations of the change in length from References 27,32 it can be said that cementite precipitation occurs in the carbon-supersaturated austenite and consequently the surrounding austenite is carbon-depleted. This process is accompanied by a contraction in length.

Dilatometry curves corresponding to the four alloys during partitioning at $600{ }^{\circ} \mathrm{C}$ for 300 seconds are shown in Figure 5(c). All alloys show a continuous and strong contraction during partitioning. Usually, carbide precipitation or the formation of austenite is related to a decrease in the length of the dilatometry samples. SEM micrographs of $M n, M n N i$ and $M n S i N i$ alloys indicate no strong carbide precipitation but a strong distribution of retained austenite films/islands. Based on the observations on phase fractions and SEM micrographs, the contraction observed during partitioning at $600{ }^{\circ} \mathrm{C}$ could be related to austenite formation by austenite reverse transformation (ART). For the $M n S i$ alloy, the contraction rate slows down after 50 seconds and is followed by a plateau indicating that the $M n S i$ alloy exhibits the same behavior as at $500{ }^{\circ} \mathrm{C}$. This dilation behavior can be interpreted as related to the precipitation of carbides in austenite. The SEM micrograph also shows pearlite formation. 
QP400-3600
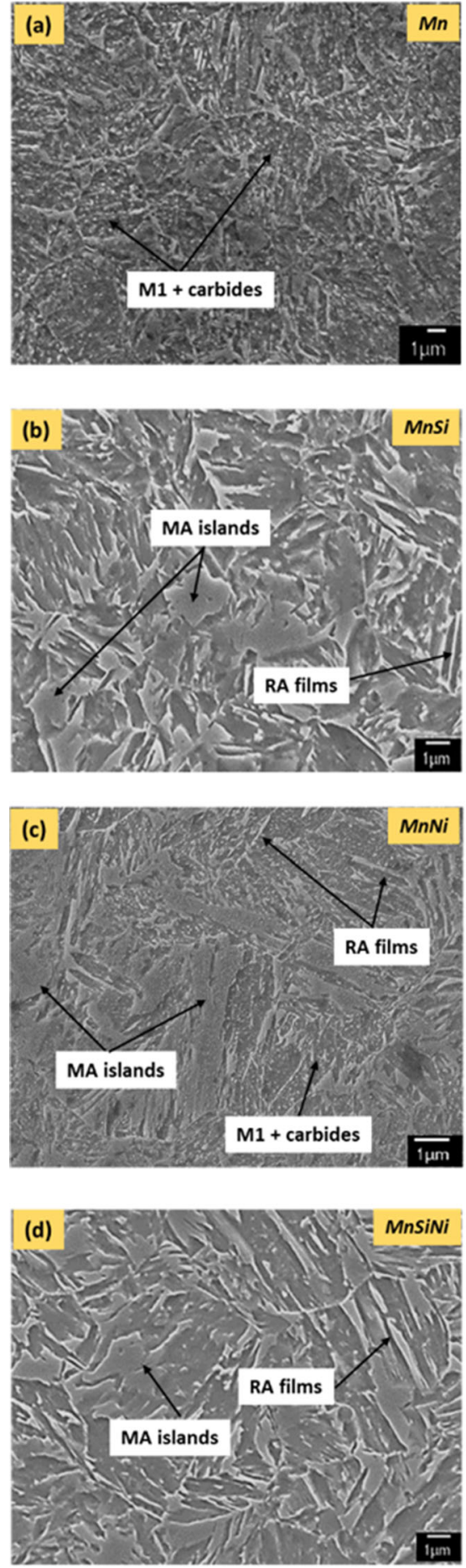

QP500-900
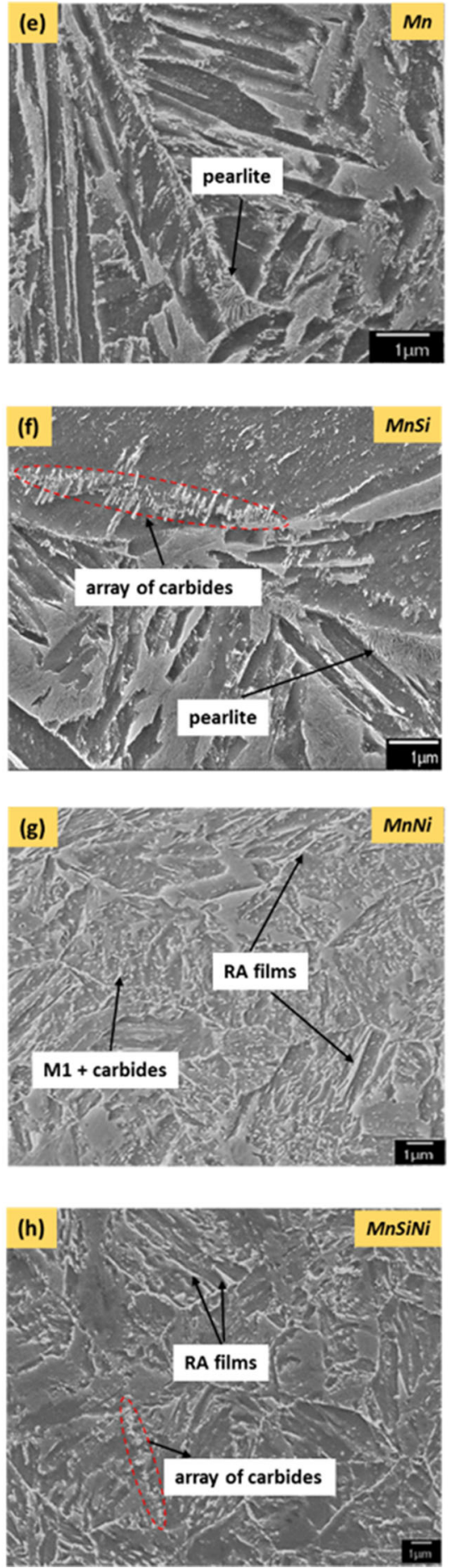

QP600-300
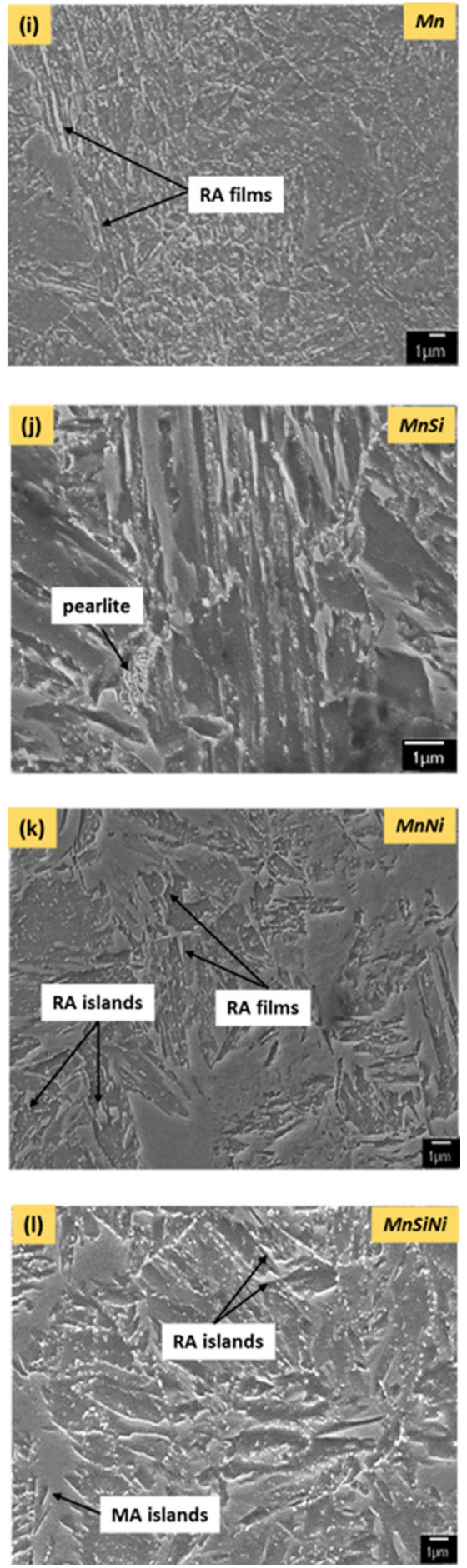

Fig. 5-Figures from left to right show SEM micrographs of Mn, MnSi, MnNi and MnSiNi alloys after QP400-3600 ( $a$ to $d$ ), QP500-900 (e to $h$ ) and QP600-300 ( $i$ to $l$ ) heat treatments. M1: primary martensite; MA: martensite/austenite islands; RA: retained austenite.

\section{Estimation of austenite reversion}

Analysis of phase fractions, SEM micrographs and dilatometry curves indicates austenite reversion in $\mathrm{Mn}$, $\mathrm{MnNi}$ and $\mathrm{MnSiNi}$ alloys during partitioning at $600{ }^{\circ} \mathrm{C}$. To estimate the fraction of reverted austenite at $600{ }^{\circ} \mathrm{C}$, a set of re-heating experiments was designed as shown in
Figure 7. First, microstructures consisting of $0.75 / 0.25$ martensite/austenite fractions were created by full austenitization and quenching to the initial quench temperature $\left(T_{\mathrm{Q}}\right)$, as in the earlier $\mathrm{Q} \& \mathrm{P}$ thermal routes shown in Figure 2. Thereafter, specimens were reheated to the respective austenitization temperatures (heating 2) 


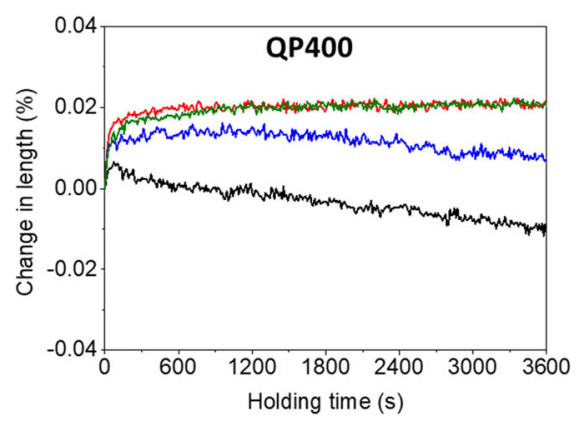

(a)

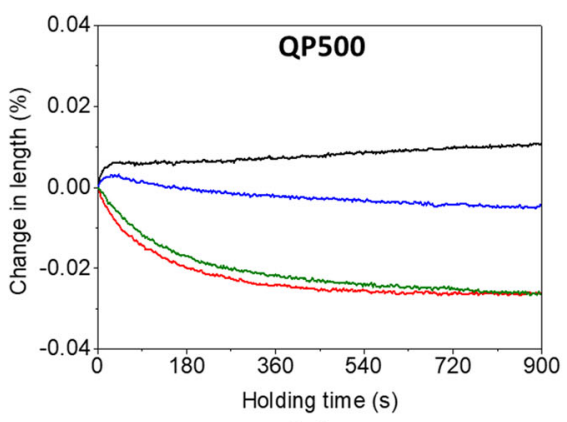

(b)

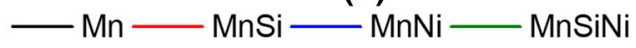

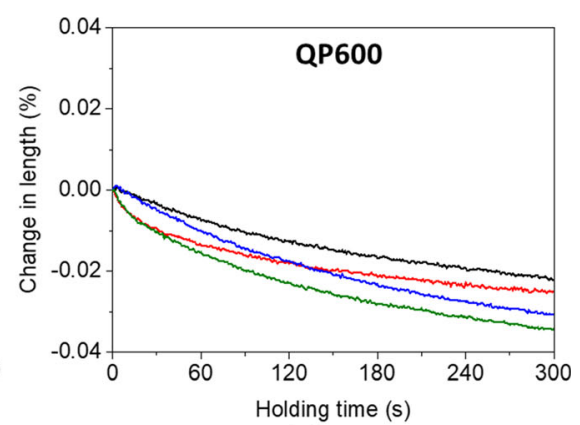

(c)

Fig. 6-Relative change in length against partitioning time after (a) QP400-3600, (b) QP500-900 and (c) QP600-300 heat treatments in Mn, $\mathrm{MnSi}, \mathrm{MnNi}$ and $\mathrm{MnSiNi}$ alloys.

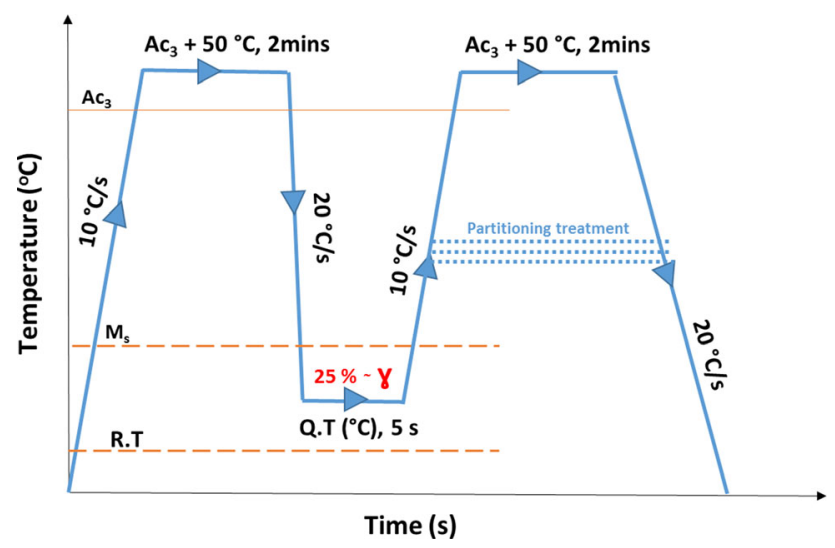

Fig. 7-Quenching and re-heating cycles applied on all four alloys to determine austenite formation temperatures during reheating.

and quenched down to room temperature after isothermally holding for 120 seconds. The methodology to determine the austenite formation temperature during reheating and the estimation of the austenite volume fraction formed during isothermal holding at $600{ }^{\circ} \mathrm{C}$ is explained below for $M n$ alloy.

The relative changes in length (solid line) and the first derivative of change in length with respect to temperature (dashed lines) during the quenching and re-heating heat treatment of all four alloys are shown in Figures 8(a) through (d). The initiation of austenite formation during heating is indicated by a drop in the respective derivative curves (dashed lines). In all the alloys, the austenite formation temperature during reheating is lower than the measured $\mathrm{Ac}_{1}$ temperature. The reason for the onset of austenite reverse transformation at temperatures lower than $\mathrm{Ac}_{1}$ could be due to the presence of pre-existing austenite before the reheating. This can influence the subsequent austenite formation during the partitioning stage since it can grow more easily because no new nucleation is needed. ${ }^{[20,33,34]}$ As Figure 8 shows, the austenite formation temperature during the reheating cycle in all four alloys is around 605 ${ }^{\circ} \mathrm{C}, 650{ }^{\circ} \mathrm{C}, 580{ }^{\circ} \mathrm{C}$ and $610^{\circ} \mathrm{C} \pm 5^{\circ} \mathrm{C}$ in the $M n, M n S i$, $\mathrm{MnNi}$ and $\mathrm{MnSiNi}$ alloy, respectively. Except for $\mathrm{MnSi}$ alloy, the rest of the alloys indicate austenite formation at temperatures very close to $600{ }^{\circ} \mathrm{C}$. This also confirms that the behavior of the dilatometry curves during partitioning at $600{ }^{\circ} \mathrm{C}$ (Figure 6(c)) in $M n, M n N i$ and $\mathrm{MnSiNi}$ alloys is related to austenite formation.

The volume fraction of austenite formed during the partitioning stage of QP600-300 heat treatment is calculated by comparing the change in length associated to its formation observed at $600{ }^{\circ} \mathrm{C}$ and the one derived from the complete BCC-to-FCC transformation. The relative change in length corresponding to complete austenite formation at $600{ }^{\circ} \mathrm{C}$ is extracted from the re-heating curve. Notably, the re-heating curve displays the transformation of only 0.75 volume fraction of $\mathrm{BCC}$ into FCC. From the comparison, the volume fraction of austenite formed after 300 seconds of partitioning at 600 ${ }^{\circ} \mathrm{C}$ is calculated to be around $0.04,0.06$ and 0.06 in $\mathrm{Mn}$, $\mathrm{MnNi}$ and $\mathrm{MnSiNi}$ alloys, respectively. A discrepancy of 1 to 2 pct can be expected because of the assumption that the kinetics of austenite formation during partitioning at $600{ }^{\circ} \mathrm{C}$ and reheating are considered to be the equivalent.

\section{DISCUSSION}

Table III shows the summary of various phenomena observed during the partitioning stage of the respective Q\&P treatments in all four alloys. With a change in the composition of the material, the microstructural events are either hindered or promoted. Hence, this section deals with understanding the influence of the alloying elements on the microstructural events during the partitioning stage and on austenite stability.

\section{A. Assessment of Carbon Partitioning}

Carbon is a key alloying element in the stabilization of austenite at room temperature in the Q\&P process. For this reason, to maximize the amount of carbon partitioned from martensite into austenite, it is of prime importance to inhibit carbon-consuming phenomena such as precipitation of carbides in primary martensite and austenite and pearlite formation during the partitioning stage. 


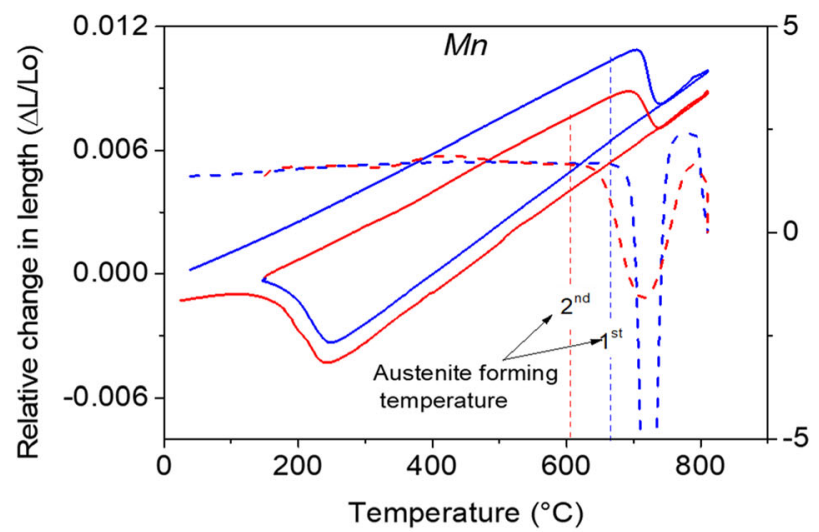

(a)

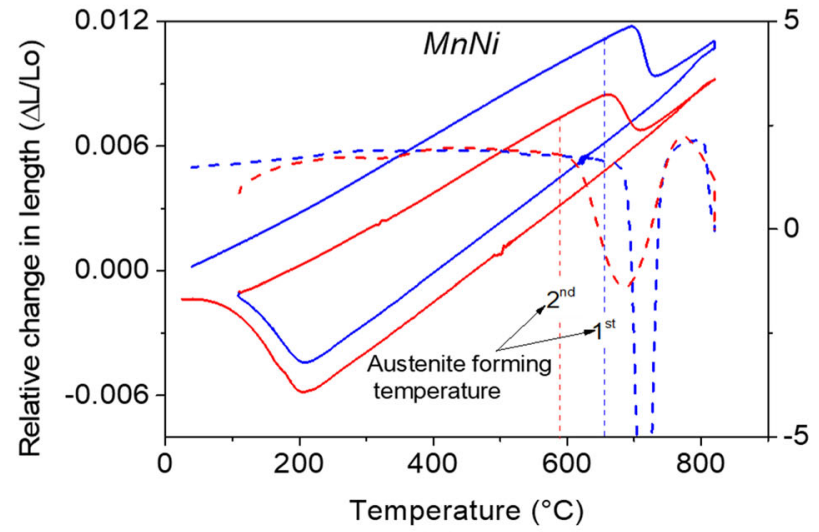

(c)

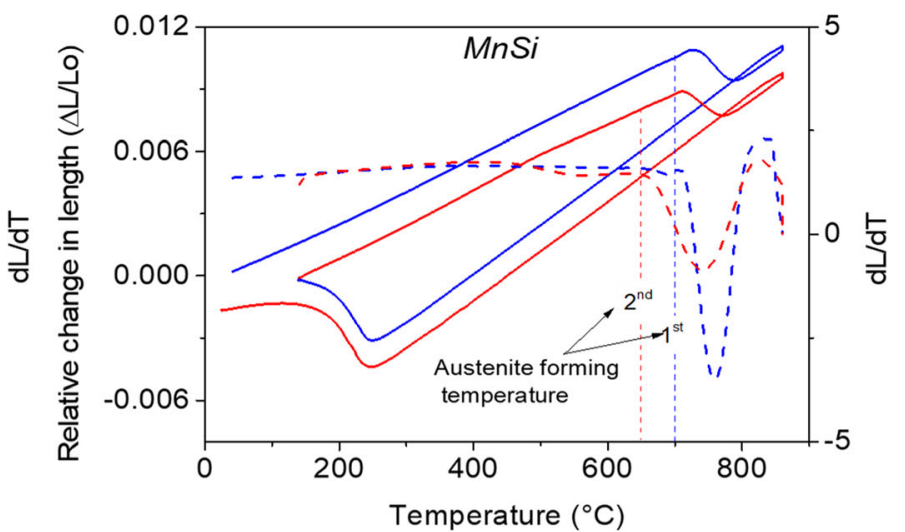

(b)

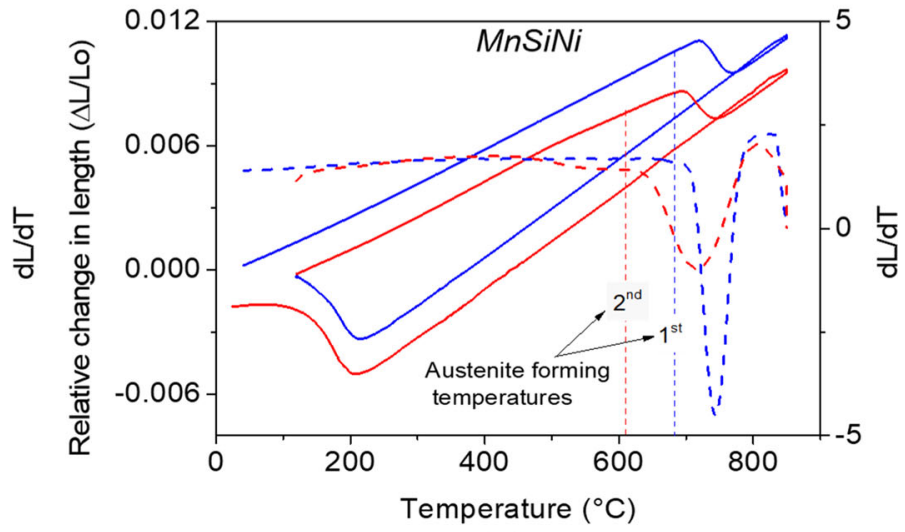

(d)

\section{- Heating 1 - Heating 2 --dL/dT 1 ---dL/dT2}

Fig. 8- Change in length during quenching and reheating heat treatment (solid line) and respective derivative curves (dashed lines) showing temperatures of austenite formation during the first and second heating cycles in (a) Mn, (b) MnSi, (c) MnNi and (d) MnSiNi alloys.

Table III. Summary of Phenomena Occurring During the Partitioning Stage of QP400-3600, QP500-900 and QP600-300 Heat Treatments in All Four Alloys

\begin{tabular}{|c|c|c|c|c|c|}
\hline \multirow[b]{2}{*}{ Heat Treatment } & \multirow[b]{2}{*}{ Alloy } & \multicolumn{4}{|c|}{ Phenomenon } \\
\hline & & $\begin{array}{l}\text { Carbide Precipitation } \\
\text { in Primary Martensite }\end{array}$ & $\begin{array}{l}\text { Carbide Precipitation } \\
\text { in Austenite }\end{array}$ & Pearlite & $\begin{array}{c}\text { Austenite Reverse } \\
\text { Transformation }\end{array}$ \\
\hline \multirow[t]{4}{*}{ QP400-3600 } & $M n$ & $\checkmark$ & - & - & - \\
\hline & $\mathrm{MnSi}$ & - & - & - & - \\
\hline & $M n N i$ & $\checkmark$ & - & - & - \\
\hline & $M n S i N i$ & - & - & - & - \\
\hline \multirow[t]{4}{*}{ QP500-900 } & $M n$ & $\checkmark$ & - & $\checkmark$ & - \\
\hline & $\mathrm{MnSi}$ & - & $\checkmark$ & $\checkmark$ & - \\
\hline & $M n N i$ & $\checkmark$ & - & - & - \\
\hline & $M n S i N i$ & - & $\checkmark$ & $\checkmark$ & - \\
\hline \multirow[t]{4}{*}{ QP600-300 } & $M n$ & $\checkmark$ & - & - & $\checkmark$ \\
\hline & $M n S i$ & $\checkmark$ & $\checkmark$ & $\checkmark$ & - \\
\hline & $M n N i$ & $\checkmark$ & - & - & $\checkmark$ \\
\hline & $M n S i N i$ & - & - & - & $\checkmark$ \\
\hline
\end{tabular}

To assess the fraction of carbon partitioned from martensite to austenite during the partitioning stage of Q\&P heat treatments the following carbon balance is considered:

$$
f_{\gamma\left(t_{p}\right)} \cdot x_{c\left(t_{p}\right)}^{\gamma}=f_{R A} \cdot x_{C}^{R A}+f_{M 2} \cdot x_{C}^{M 2}
$$


where $f_{\gamma}, f_{\mathrm{M} 2}, f_{\mathrm{RA}}$ and $x_{c}^{\gamma}, x_{C}^{M 2}, x_{C}^{R A}$ are the volume fractions and carbon contents of austenite at the end of the partitioning stage, fresh martensite and retained austenite, respectively. $t_{p}$ is the partitioning time. This implies that the carbon content present in the austenite at the end of the partitioning process is a summation of the carbon content of retained austenite and the secondary martensite at the end of the heat treatment. $f_{\mathrm{RA}}$ and $f_{M 2}$ are measured by x-ray diffraction and dilatometry, respectively. $x_{C}^{R A}$ is measured from the lattice parameter as explained in Section II-A. The use of an empirical equation to calculate the carbon content of fresh martensite $\left(x_{C}^{M 2}\right)$ from the martensite start temperature implies knowing the concentration of substitutional alloying elements in the retained austenite, as they are considered to play a crucial role in austenite stabilization. However, the concentrations of substitutional alloying elements (manganese, nickel and silicon) in austenite are not assessed in this work. Hence, the carbon content in austenite by the end of partitioning stage is calculated by assuming two extreme cases, that is, assuming that fresh martensite has a carbon content: (1) similar to that of retained austenite-upper limit and (2) similar to that of the nominal composition (0.19 wt pct) of the material, which implies an absence of carbon partitioning in the austenite regions that became M2 on quenching-lower limit. The precise amount of carbon in fresh martensite will be in between these upper and lower limits.

Figures 9(a) through (c) shows the range of total enrichment of carbon in the austenite at the end of partitioning stage of QP400-3600, QP500-900 and QP600-300 heat treatment, respectively. Note that the word "total" in this context indicates the product of the local carbon concentration of austenite and the volume fraction of austenite. In Figure 9, the black dashed line indicates the total carbon in austenite at the beginning of the partitioning step, calculated as the product of nominal carbon content $(0.19 \mathrm{wt} \mathrm{pct})$ and the initial fraction of austenite ( 0.25$)$. The red dashed line indicates the carbon content of austenite at the end of the partitioning step assuming complete carbon partitioning from martensite (depleted to an equilibrium carbon composition, $0.013 \mathrm{wt} \mathrm{pct}$ ) into austenite at the end the partitioning step. As expected, the upper and lower limits for the total carbon in austenite at the end of the partitioning step lie in between the black and red lines.

The fraction of carbon remaining in primary martensite in either solid solution and/or carbides is estimated by carbon balancing.

$$
\bar{x}=f_{\gamma\left(t_{p}\right)} \cdot x_{c\left(t_{p}\right)}^{\gamma}+f_{M 1\left(t_{p}\right)} \cdot x_{c\left(t_{p}\right)}^{M 1}+f_{p\left(t_{p}\right)} \cdot x_{c\left(t_{p}\right)}^{p}
$$

where $\bar{x}$ is the total fraction of carbon present in the alloy $(0.19 \mathrm{wt} \mathrm{pct}), f_{i}$ and $x_{c}^{i}$ represent the volume fraction and carbon content of phase $i$, being $i=$ austenite $(\gamma)$, primary martensite $(\mathrm{M} 1)$ and pearlite $(\mathrm{P})$, at the end of isothermal holding. It should be noted that the fraction of carbon in M1 is the summation of carbon present in solid solution and the fraction of carbon in the form of carbides. Equation [4] is modified accordingly depending on the microstructural constituents present during partitioning at different temperatures to evaluate the carbon remaining in primary martensite at the end of the isothermal holding at every partitioning temperature.

\section{B. Interplay of Alloying Elements at Different Partitioning Temperatures}

\section{Partitioning at $400{ }^{\circ} \mathrm{C}$}

In alloys without silicon ( $M n$ and $M n N i)$, carbide precipitation is observed to be significant compared to alloys with silicon ( $\mathrm{MnSi}$ and $\mathrm{MnSiNi}$ ), which could be due to carbide precipitation during the initial quench (auto-tempering) ${ }^{[35]}$ or the partitioning stage. ${ }^{[17]}$ The work of Reference 36 reports that silicon can retard cementite formation kinetics, but transitional carbides can still be observed in primary martensite. Hence, it is highly probable that the carbides observed in $\mathrm{MnSi}$ and $\mathrm{MnSiNi}$ alloys are transitional carbides. The equilibrium phase diagram, calculated using Thermo-Calc, shows that in $M n$ and $M n N i$ alloys, cementite, $\mathrm{M}_{23} \mathrm{C}_{6}$ and $\mathrm{M}_{5} \mathrm{C}_{2}$ types of carbides are expected to form. Since these are equilibrium phases, they do not dissolve with the increase in partitioning time. This is why even after a partitioning time of 3600 seconds, carbides are clearly evident in the respective SEM micrographs.

In alloys without silicon, formation of cementite or stable carbides reduces the fraction of carbon in solid solution in M1. Thus, a lower fraction of carbon is available to partition into the austenite phase during the partitioning stage. Eventually, at the end of the partitioning stage in $M n$ and $M n N i$ alloys, austenite is enriched with lower carbon content (Figure 4(a)).

Figure 9(a) shows that complete carbon partitioning from martensite to austenite did not occur in any of the alloys (all points are below the red dashed line). This indicates that a significant fraction of carbon remains in the martensite either in solid solution or in the form of carbides, even in alloys with silicon ( $\mathrm{MnSi}$ and $\mathrm{MnSiNi}$ ), which showed negligible carbide precipitation (Figures 5(b) and (d)). Assuming full partitioning and using Eq. [4], the total amount of carbon remaining in primary martensite is calculated to be around $0.05 \mathrm{wt}$ pet C. HajyAkbari et al. ${ }^{[35]}$ observed similar carbon contents in primary martensite from 3D-APT measurements in a $0.3 \mathrm{C}-1.6 \mathrm{Si}-3.5 \mathrm{Mn}$ (wt pct) steel after partitioning at $400{ }^{\circ} \mathrm{C}$ for 200 seconds. A reason for this could be that some of the carbon is trapped in the defects/dislocations of the primary martensite. ${ }^{[37]}$ Moreover, due to their very small size, some transitional carbides formed during the initial quench may not be visible in the SEM micrographs. ${ }^{[38]}$ These factors can be the reason for the presence of $0.05 \mathrm{wt}$ pct carbon in primary martensite. In alloys without silicon, $M n$ and $\mathrm{MnNi}$, in which significant carbide precipitation is observed, this fraction is around 0.09 and 0.10 wt pet $C$, respectively. Hence, it can be interpreted that in $M n$ and $\mathrm{MnNi}$ alloys at least 0.04 and 0.05 wt pet $\mathrm{C}$ of the total carbon is consumed by carbide precipitation in primary martensite during the partitioning stage, respectively. 


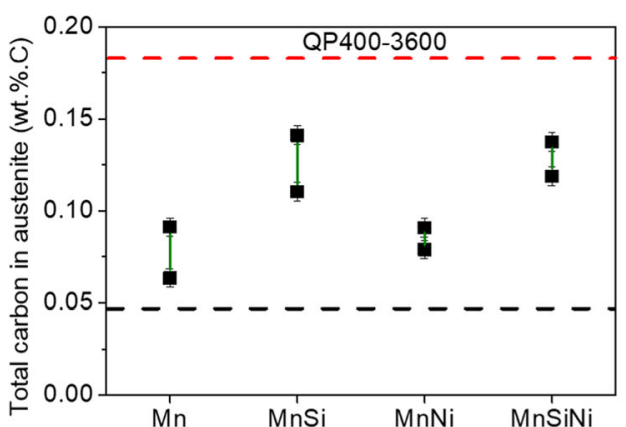

(a)

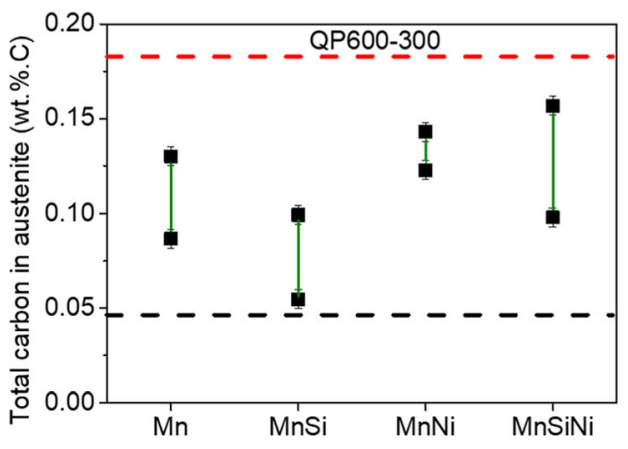

(c)

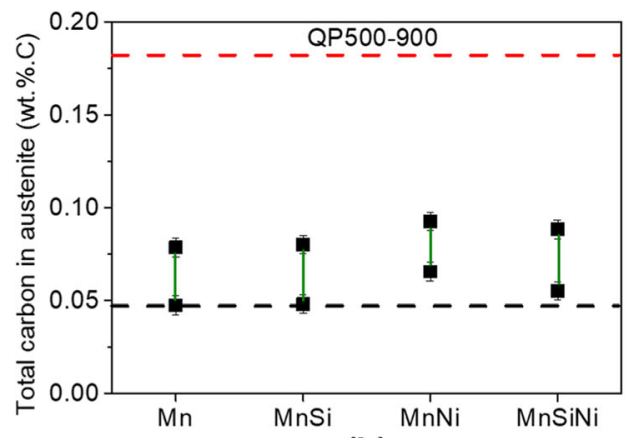

(b)

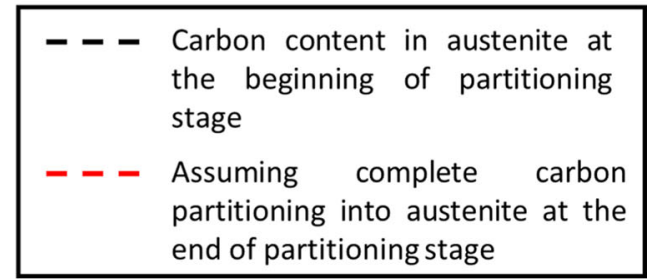

Fig. 9-Upper and lower limits of total carbon present in austenite phase at the end of the partitioning step (black dots) in the four alloys. Note that these values represent the product of the local carbon concentration in austenite and the volume fraction of austenite. $(a)$ QP400-3600, $(b)$ QP500-900 and (c) QP600-300.

These calculations also suggest that there is more carbide precipitation in $M n N i$ than $M n$ alloy.

Despite the presence of a significant fraction of carbides within primary martensite and less carbon enrichment of the austenite, a high fraction of austenite is retained in the alloy with nickel $(M n N i)$. This can be explained by the austenite stabilizing ability of nickel. ${ }^{[20 \text {, }}$ 39-41] Comparison of all four alloys shows that the fraction of retained austenite after both 300 and 3600 seconds of holding is in the order of $M n<M n S i<$ $M n N i<M n S i N i$. This also shows that, during the partitioning stage of QP400 heat treatment, addition of either silicon or nickel helps in austenite stabilization by either suppressing carbide formation or increasing the thermal stability of austenite, respectively. These mechanisms are kept active with the increase of the isothermal holding time. The combination of suppression of carbide precipitation due to the presence of silicon and the ability of nickel to increase thermal stability of austenite in $M n S i N i$ alloy results in the retention of the largest fraction of austenite for the QP400 condition.

\section{Partitioning at $500{ }^{\circ} \mathrm{C}$}

In contrast to isothermal holding at $400{ }^{\circ} \mathrm{C}$, a decrease in the fraction of retained austenite with an increase in partitioning time is observed in all four alloys. In their recent work, Hidalgo et al. ${ }^{[1]}$ showed carbon redistribution between martensite and austenite in a 0.31C-4.58Mn-1.5Si (wt pct) steel at a partitioning temperature of $500{ }^{\circ} \mathrm{C}$, using DICTRA simulations. It is observed that, in $<1$ seconds, thin film-like austenite is enriched with $1.50 \mathrm{wt}$ pct carbon. Hence, austenite grains are enriched with a high fraction of carbon already at the very beginning of the partitioning stage. In the present study, precipitation of carbides and/or the formation of pearlite in the already carbon-enriched austenite is observed with the increase of holding time during partitioning at $500{ }^{\circ} \mathrm{C}$. These phenomena consume carbon thereby reducing the carbon available to homogenize in the rest of the austenite grains. ${ }^{[27]}$ Hence, during the final quench, the austenite that is deficient in carbon will transform into fresh martensite. This explains the decreasing trend of the retained austenite volume fraction with an increase in isothermal holding time observed in all four alloys.

However, in $M n N i$ alloy, pearlite and carbides in austenite films are not observed. This is due to the absence of silicon in the material, which leads to a promotion of carbide precipitation in martensite compared with the other alloys. Moreover, the presence of nickel hinders pearlite formation by shifting the nose of pearlite to lower and much longer isothermal holding times. ${ }^{[42]}$

Sections of the TTT diagrams of all the four alloys (Figure 10), corresponding to the ferrite/ pearlite transformation fronts calculated using MAP_STEEL_MUCG83, ${ }^{[43]}$ show that the partitioning temperature of $500{ }^{\circ} \mathrm{C}$ is close to the nose of pearlite in all alloys except for $M n N i$ alloy. However, the predicted isothermal holding times are far from the current experimental times. To investigate this, a set of experiments was performed by cooling down completely 
austenitic samples to $500{ }^{\circ} \mathrm{C}$ and isothermally holding them for 1800 seconds. Interestingly, no pearlite formation was observed in either of the alloys. This suggests that the presence of martensite-assisted pearlite formation through rapid carbon enrichment of austenite promoted at this partitioning temperature.

The SEM micrographs of Q\&P-treated samples of $M n, M n S i$ and $M n S i N i$ alloys (Figures 5(e), (f) and (h)) show that pearlite formation is initiated along the grain boundaries of martensite and prior austenite. According to the classical nucleation theory, the nucleation rate of carbides is high at the regions where the precipitation driving force is high and activation energy for carbide nucleation is low. ${ }^{[4]}$ Both conditions are fulfilled at the martensite/austenite interfaces right after carbon partitioning. These interfaces are potential nucleation sites for carbide precipitation that initiate pearlite formation. It is calculated that around 0.02 to $0.03 \mathrm{wt}$ pet of the total carbon is consumed by pearlite.

Figure 9(b) shows the maximum and minimum range of total carbon content partitioned into austenite during the partitioning stage of QP500-900 heat treatment. Based on Figure 9(b) and the above analysis, it can be interpreted that even considering maximum partitioning of carbon, around 0.08 to 0.09 wt pet of total carbon is left out in the primary martensite of $M n, M n S i$ and $M n S i N i$ alloys. Assuming that the 0.75 volume fraction of primary martensite has an equilibrium composition ( 0.013 wt pct), only $0.01 \mathrm{wt}$ pct of total carbon is in the form of solid solution. The rest of the carbon is in the form of carbides in primary martensite. Hence, the maximum fraction of total carbon in primary martensite seems to be consumed by the carbides precipitated along the martensite/austenite interfaces that are evident from the SEM micrographs. It can be interpreted that, by the end of the partitioning stage at $500{ }^{\circ} \mathrm{C}$, competitive reactions such as carbide precipitation in primary martensite and/or pearlite consumed the maximum fraction of total carbon.

Comparison of all four alloys shows that the volume fraction of retained austenite at the end of both QP500-180 and QP500-900 heat treatments follows the order $M n<M n S i<M n S i N i<M n N i$ indicating that

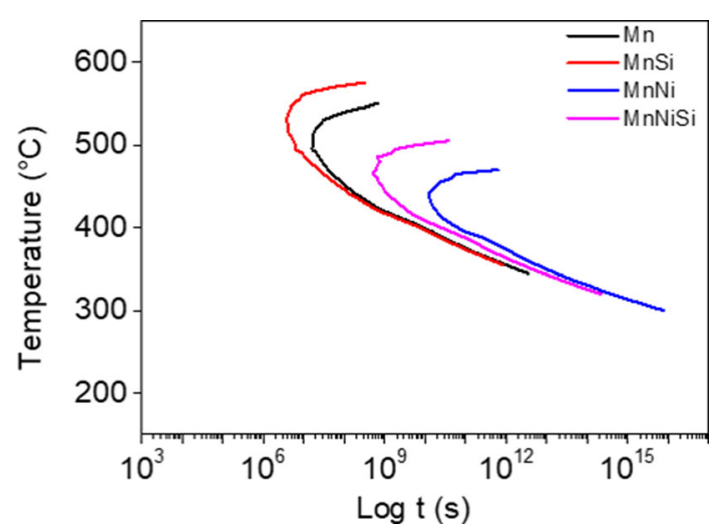

Fig. 10-Section of the TTT diagram calculated using MAP_STEEL_MUCG83 showing the nose of ferrite/pearlite formation in $\bar{M} n, M n S i, M n N i$ and $M n S i N i$ alloys. nickel has a positive impact on the thermal stability of retained austenite. Addition of silicon reduces the chances of carbide precipitation in martensite, leading to precipitation of carbides in austenite and pearlite formation during partitioning. These effects reduce the stabilizing effect of manganese and nickel. In summary, suppression of silicon (to promote carbide precipitation in primary martensite instead of in austenite) and addition of nickel (to suppress pearlite formation) along with holding times $<180$ seconds seem beneficial to prevent competitive reactions during partitioning treatments at temperatures that are close to the pearlite nose in the TTT diagrams.

\section{Partitioning at $600{ }^{\circ} \mathrm{C}$}

In $\mathrm{Mn}, \mathrm{MnSi}$ and $\mathrm{MnSiNi}$ alloys, no major competitive phenomena are observed during the isothermal holding at $600{ }^{\circ} \mathrm{C}$. Instead, an increase in the retained austenite fraction with partitioning time is observed, which is due to austenite reverse transformation (ART). Figure 9(c) shows that the total amount of carbon accommodated by austenite is higher compared to that at $400{ }^{\circ} \mathrm{C}$ and $500{ }^{\circ} \mathrm{C}$, which is due to austenite reversion during the partitioning stage.

The analysis in Section III indicates that, irrespective of the different alloying elements, $\mathrm{MnNi}$ and $\mathrm{MnSiNi}$ alloys show similar volume fractions of newly formed austenite after partitioning at $600{ }^{\circ} \mathrm{C}$ for 300 seconds. To understand the influence of alloying elements on the kinetics of austenite reverse transformation at $600{ }^{\circ} \mathrm{C}$, 1D simulations of carbon partitioning from martensite to austenite in $M n, M n N i$ and $M n S i N i$ alloys are carried out using DICTRA software ${ }^{[45]}$ using the TCFE8 and MobFe3 database. Earlier observations ${ }^{[46-48]}$ showed that the martensitic lath widths are frequently found to be on the order of $0.2 \mu \mathrm{m}$. Hence, martensite and austenite lath sizes are defined to be 200 and $66 \mathrm{~nm}$, respectively. This corresponds to 0.75 and 0.25 volume fractions of martensite and austenite, respectively, representing the outset conditions of the partitioning stage of Q\&P experiments. Because of the mirror boundary conditions in the calculation domain in DICTRA, only the half thickness of the lath width is considered.

Figure 11 shows the kinetics of austenite reverse transformation (interface movement towards martensite) in $M n, M n N i$ and $M n S i N i$ alloys calculated with DICTRA. For all three alloys, the transformation starts at the very beginning of isothermal holding. In all three alloys, at around $10^{-4}$ seconds, a shift from the NPLE to PLE mode of transformation ${ }^{[20]}$ is observed. It can be observed that the kinetics of austenite transformation are faster in alloys with nickel. Although $M n N i$ alloy shows a relatively faster kinetics than $M n S i N i$ alloy, after 300 seconds of partitioning time the distance moved by the interface is similar in both the alloys. Comparison between $M n$ and $M n N i$ alloys shows that, while the addition of both manganese and nickel assists austenite growth, the austenite reverse kinetics are faster in the presence of nickel. Comparing alloys $M n N i$ and $\mathrm{MnSiNi}$ shows that alloys without silicon exhibit a faster kinetics of austenite reversion during the early stages of 


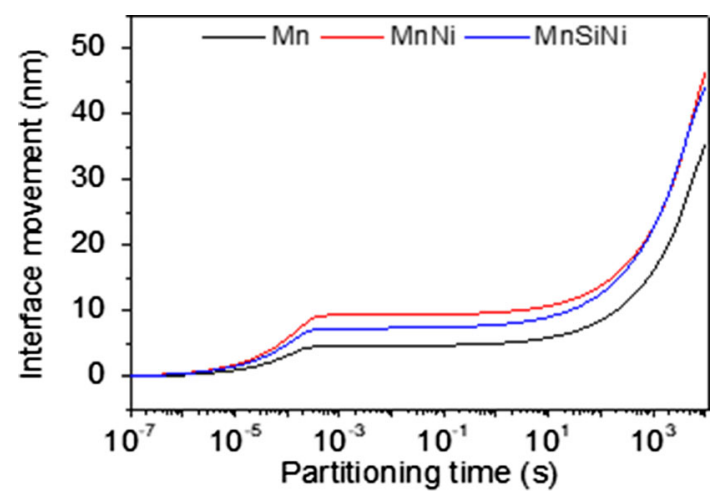

Fig. 11-Austenite reversion kinetics in $\mathrm{Mn}, \mathrm{MnNi}$ and $\mathrm{MnSiNi}$ alloys represented as the interface position versus isothermal holding time at a partitioning temperature of $600{ }^{\circ} \mathrm{C}$, calculated using Dictra. Position " 0 " defines the location of the martensite/austenite interface at the beginning of the partitioning step and before austenite reversion starts.

isothermal holding. As silicon is a strong ferrite stabilizer, it retards austenite formation kinetics, which results in a sluggish austenite reversion at the beginning of isothermal holding in $\mathrm{MnSiNi}$ alloy. ${ }^{[49,50]}$

$\mathrm{MnSiNi}$ alloy displays a similar fraction of reverted austenite at the end of the partitioning stage and a higher carbon content of retained austenite compared with $\mathrm{MnNi}$ alloy. However, the volume fraction of retained austenite is lower in the final microstructure of $\mathrm{MnSiNi}$ alloy. Figure 12 shows that the martensite starting temperature of $\mathrm{MnSiN} i$ alloy is higher than that of $M n N i$ alloy. This could be because $M n N i$ alloy contains 0.5 wt pct higher nickel concentration than $\mathrm{MnSiNi}$ alloy. Moreover, the work of Wang et al., ${ }^{[51]}$ based on the phenomenological results, reported that, although silicon and manganese alone decrease the martensite starting temperature $\left(M_{\mathrm{s}}\right)$, the interaction of silicon-manganese has an increasing effect on the $M s$ temperature. However, further research is required to understand the impact of the interaction of alloying elements on the $M_{\mathrm{s}}$ temperature.

Observations of $\mathrm{Mn}, \mathrm{MnNi}$ and $\mathrm{MnSiNi}$ alloys show that the fraction of retained austenite rises with partitioning time while that of fresh martensite remains almost the same. This indicates that reverted austenite is more stable during the final quench than in the pre-existing austenite. This could be explained by considering the formation mechanism of reverted austenite, which involves local enrichment in manganese and nickel, as reported by Ding et al. ${ }^{[20]}$ Therefore, suppression of silicon and addition of nickel ( $\mathrm{MnNi}$ alloy), along with holding times of up to 300 seconds at $600{ }^{\circ} \mathrm{C}$, seem beneficiary for the stabilization and maximization of the retained austenite volume fraction in the final microstructure.

In summary, the interplay between the substitutional alloying elements manganese, silicon and nickel with carbon have either a positive or negative influence on the Q\&P microstructural development through changes in the carbon partitioning and stabilization of austenite depending on the partitioning temperature. At conventional partitioning temperature $\left(400{ }^{\circ} \mathrm{C}\right)$, alloys

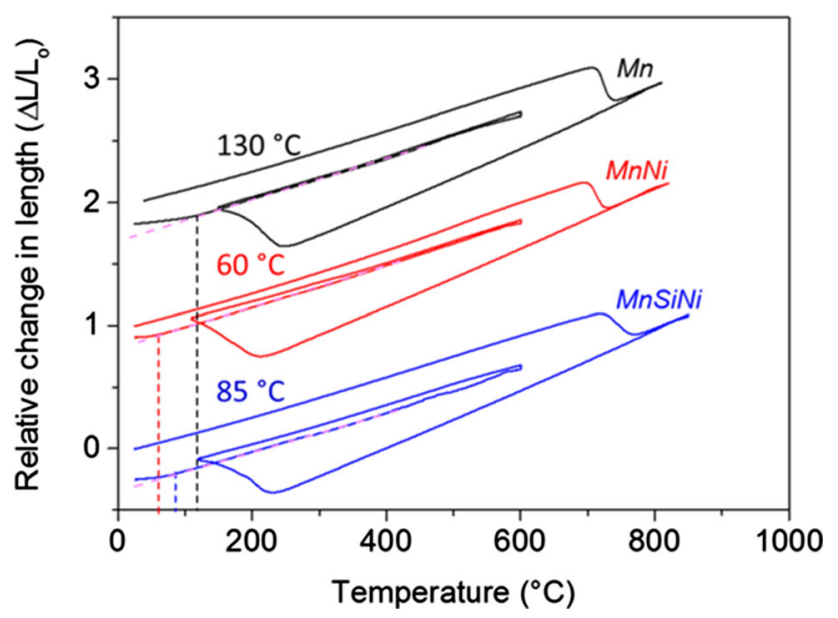

Fig. 12-Dilatometry curves of $\mathrm{Mn}, \mathrm{MnNi}$ and $\mathrm{MnSiNi}$ alloys indicating the martensite starting temperature $\left(M_{\mathrm{s}} \pm 5{ }^{\circ} \mathrm{C}\right)$ during the final quench of QP600-300 heat treatment.

containing silicon significantly promote austenite stability, which is even significantly enhanced with the addition of nickel. At temperatures close to the nose of pearlite formation $\left(500{ }^{\circ} \mathrm{C}\right)$, alloys with nickel and without silicon, partitioned for shorter holding times $(<$ 180 seconds), are recommended to retain higher fractions of austenite. It seems that austenite reverse transformation during the partitioning at $600{ }^{\circ} \mathrm{C}$ is beneficial, and the reverted austenite can be more stable during the final quench than the sole carbon-enriched austenite. This also shows that partitioning temperatures above and below the nose of pearlite stabilize higher fractions of austenite, whereas the fraction of retained austenite increases with increasing partitioning time. The addition of nickel to a Q\&P steel alloy is key in creating a controlled Q\&P microstructure as it can assist in controlling major competing reactions, such as precipitation of carbides inside austenite and pearlite formation, and in increasing thermal stability of austenite facilitating high temperature $\mathrm{Q} \& \mathrm{P}$ cycles.

\section{CONCLUSIONS}

Four different medium manganese steels were investigated to understand the impact of silicon and nickel on the carbon partitioning, microstructural evolution and stability of retained austenite during quenching and partitioning processing at $400{ }^{\circ} \mathrm{C}, 500{ }^{\circ} \mathrm{C}$ and $600{ }^{\circ} \mathrm{C}$ for different times. The main conclusions drawn from the current work are:

- After partitioning at $400{ }^{\circ} \mathrm{C}, 500{ }^{\circ} \mathrm{C}$ and $600{ }^{\circ} \mathrm{C}$ for up to 3600 seconds, a significant fraction of carbon is not partitioned into austenite but remains in primary martensite either in solid solution or in the form of carbides.

- Partitioning at $400{ }^{\circ} \mathrm{C}$ : the presence of nickel decreases the carbon content required to retain austenite at room temperature, which results in higher fractions of retained austenite compared to 
alloys without nickel. The addition of silicon prevents cementite precipitation, making more carbon available for partitioning. Thus, in the presence of both nickel and silicon significant fractions of austenite are stabilized at room temperature.

- Partitioning at $500{ }^{\circ} \mathrm{C}$ : pearlite formation is prevented as the addition of nickel shifts the nose of pearlite to longer times. Also, the absence of silicon ( $\mathrm{Mn}$ and $\mathrm{MnNi}$ ) promotes carbide precipitation in primary martensite thereby avoiding supersaturation of austenite grains during the isothermal holding, contributing to the control of competitive reactions during the partitioning stage.

- Partitioning at $600{ }^{\circ} \mathrm{C}$ : the presence of nickel increases the austenite reversion kinetics, while silicon hinders it. To retain a higher volume fraction of austenite, austenite reversion during partitioning seems to be beneficial as the reverted austenite during the final quench is more stable than the pre-existing austenite.

- At all partitioning temperatures, except at $500^{\circ} \mathrm{C}$, an increase in partitioning time results in an increase in the volume fraction of retained austenite.

The results of the current research work provide an in-detail analysis of the impact of alloying elements on the microstructural evolution and austenite stability of medium manganese Q\&P steels. Addition of nickel and adjustments of the Q\&P cycles to promote the formation of reverted austenite are both potential strategies for the design of novel Q\&P microstructures.

\section{ACKNOWLEDGMENTS}

The authors thank Alfonso Navarro-Lopez for his fruitful contribution during discussions. This research work has been carried out in the framework of the HighQP project (Proposal Number: 709855), funded by the Research Fund for Coal and Steel (RFCS).

\section{AUTHOR CONTRIBUTIONS}

SA: Conceptualization, investigation, formal analysis, writing-original draft, visualization. CC-C: Formal analysis, supervision, writing-review and editing. ZA: Investigation, writing-review and editing. MA: investigation, writing-review and editing. AA: Resources, writing-review and editing. JS: Supervision, writing-review and editing, funding acquisition. MJS: Conceptualization, resources, supervision, writing-review and editing, project administration, funding acquisition.

\section{DATA AVAILABILITY}

The raw data required to reproduce the findings of this research study cannot be shared at this time as the data also form part of an on-going study.

\section{OPEN ACCESS}

This article is licensed under a Creative Commons Attribution 4.0 International License, which permits use, sharing, adaptation, distribution and reproduction in any medium or format, as long as you give appropriate credit to the original author(s) and the source, provide a link to the Creative Commons licence, and indicate if changes were made. The images or other third party material in this article are included in the article's Creative Commons licence, unless indicated otherwise in a credit line to the material. If material is not included in the article's Creative Commons licence and your intended use is not permitted by statutory regulation or exceeds the permitted use, you will need to obtain permission directly from the copyright holder. To view a copy of this licence, visit http://creat ivecommons.org/licenses/by/4.0/.

\section{REFERENCES}

1. D.K. Matlock and J.G. Speer: Microstructure and Texture in Steels, A. Haldar, S. Suwas, and D. Bhattacharjee, eds., Springer London, 2009, pp. 185-205.

2. J.G. Speer, A.M. Streicher, D. Matlock, F. Rizzo, G. Krauss: Mater. Sci. Technol. Meeting, Chicago, IL; United States, 2009.

3. S. Oliver, T.B. Jones, and G. Fourlaris: Mater. Sci. Technol., 2007, vol. 23, pp. 423-31.

4. J. Chiang, B. Lawrence, J.D. Boyd, and A.K. Pilkey: Mater. Sci. Eng. A, 2011, vol. 528 (13), pp. 4516-21.

5. J.G. Speer, F. Rizzo, D. Matlock, and D.V. Edmonds: Mater. Res., 2005, vol. 8 (4), pp. 417-23.

6. J.G. Speer, D.K. Matlock, B.C. De Cooman, and J.G. Schroth: Acta Mater., 2003, vol. 51 (9), pp. 2611-22.

7. M.J. Santofimia, L. Zhao, and J. Sietsma: Metall. Mater. Trans. A, 2008, vol. 40A (1), pp. 46-57.

8. G.A. Thomas, J.G. Speer, and D.K. Matlock: Metall. Mater. Trans. A, 2011, vol. 42A, pp. 3652-59.

9. A. Arlazarov, M. Ollat, J.P. Masse, and M. Bouzat: Mater. Sci. Eng. A, 2016, vol. 661, pp. 79-86.

10. A.S. Nishikawa, G. Miyamoto, T. Furuhara, A.P. Tschiptschin, and H. Goldenstein: Acta Mater., 2019, vol. 179, pp. 1-16.

11. J. Hidalgo, C. Celada-Casero, and M.J. Santofimia: Mater. Sci. Eng. A, 2019, vol. 754, pp. 766-77.

12. M.J. Santofimia, L. Zhao, I. Povstugar, P.P. Choi, D. Raabe, J. Sietsma: Proc 3rd int symp steel sci (ISSS 2012), N.H. Furuhara T, Ushioda K, Editor: Kyoto: The Iron and Steel Institute of Japan, 2012, vol. 155.

13. D. De Knijf, M.J. Santofimia, H. Shi, V. Bliznuk, C. Föjer, R. Petrov, and W. Xu: Acta Mater., 2015, vol. 90, pp. 161-68.

14. M. Gouné, S. Aoued, F. Danoix, G. Geandier, A.P. Quintin, J.C. Hell, M. Soler, and S.Y.P. Allain: Scr. Mater., 2019, vol. 162, pp. 181-84.

15. E.J. Seo, L. Cho, and B.C. De Cooman: Acta Mater., 2016, vol. 107, pp. 354-65.

16. Y. Toji, H. Matsuda, M. Herbig, P.P. Choi, and D. Raabe: Acta Mater., 2015, vol. 65, pp. 215-28.

17. K. Kim and S.J. Lee: Mater. Sci. Eng. A, 2017, vol. 698, pp. 183-90.

18. F. Rizzo, A.R. Martins, J.G. Speer, D.K. Matlock, A. Clarke, and B.C. De Cooman: Mater. Sci. Forum, 2007, vol. 539, pp. 4476-81.

19. D.T. Pierce, D.R. Coughlin, K.D. Clarke, E. De Moor, J. Poplawsky, D.L. Williamson, B. Mazumder, J.G. Speer, A. Hood, and A.J. Clarke: Acta Mater., 2018, vol. 151, pp. 454-69.

20. R. Ding, Z. Dai, M. Huang, Z. Yang, C. Zhang, and H. Chen: Acta Mater., 2018, vol. 147, pp. 59-69.

21. C.F. Jatczak: SAE Technical Paper, 1980, 800426.

22. D.J. Dyson and B. Holmes: J. Iron Steel Inst., 1970, vol. 208, pp. 469-74. 
23. N. Vandijk, A. Butt, L. Zhao, J. Sietsma, S. Offerman, J. Wright, and S. Vanderzwaag: Acta Mater., 2005, vol. 53 (20), pp. 5439-47.

24. L. Zhao, N.H. Van Dijk, E. Bruck, J. Sietsma, and S. Van der Zwaag: Mater. Sci. Eng. A, 2001, vol. 313 (1), pp. 145-52.

25. B.D. Cullity and C.D. Graham: Introduction to Magnetic Materials, 2nd ed., IEEE/Wiley, Hoboken, 2009.

26. A.W. El-Morsy and A.I.Z. Farahat: J. Eng. Mater. Technol., 2017, vol. 140 (2), art. no. 021005.

27. S. Ayenampudi, C. Celeda-Casero, J. Siestma, and M.J. Santofimia: Mater., 2019, vol. 8, p. 100492.

28. S. Yang and H.K.D.H. Bhadeshia: Mater. Sci. Technol., 2007, vol. 23 , pp. $556-60$.

29. H. Kim, J. Inoue, M. Okada, and K. Nagata: ISIJ Int., 2017, vol. 57 (12), pp. 2229-36.

30. C. Celada-Casero, C. Kwakernaak, J. Sietsma, and M.J. Santofimia: Mater. Des., 2019, vol. 178, p. 107847.

31. M.J. Santofimia, L. Zhao, and J. Sietsma: Mater. Sci. Forum, 2012, vols. 706-709, pp. 2290-95.

32. R. Kannan, Y. Wang, and L. Li: Metall. Mater. Trans. A, 2017, vol. 48A, pp. 948-52.

33. R. Ding, D. Tang, and A. Zhao: Scr. Mater., 2014, vol. 88, pp. 21-24.

34. R. Wei, M. Enomoto, R. Hadian, H.S. Zurob, and G.R. Purdy: Acta Mater., 2013, vol. 61 (2), pp. 697-707.

35. F. HajyAkbary, J. Sietsma, G. Miyamoto, T. Furuhara, and M.J. Santofimia: Acta Mater., 2016, vol. 104, pp. 72-83.

36. H.K.D.H. Bhadeshia and D.V. Edmonds: Metall. Trans. A, 1979, vol. 10A, pp. 895-907.

37. G. Krauss and A.R. Marder: Metall. Trans., 1971, vol. 2, pp. 2343-57.
38. A. Koniger, C. Hammerl, M. Zeitler, and B. Rauschenbach: Phys. Rev. B, 1997, vol. 55 (13), pp. 8143-47.

39. J.I. Kim and J.W. Morris: Metall. Trans. A, 1981, vol. 12A (11), pp. 1957-63.

40. S.J. Kim, C.G. Lee, T.H. Lee, and C.S. Oh: Scr. Mater., 2003, vol. 48, pp. 539-544.

41. N. Zhu, Q. Wu, Y. He, X. Lu, L. Li, and P. Hu: Steel Res. Int., 2014, vol. 85 (2), pp. 143-54.

42. D. A. Scott: Ph.D Thesis, 1947, The University of British Columbia, Canada, 1947.

43. H.K.D.H. Bhadeshia: Met. Sci., 1982, vol. 16, pp. 159-65.

44. T. Sourmail and H.K.D.H. Bhadeshia: Calphad, 2003, vol. 27, pp. 169-75.

45. A. Borgenstam, L. Hoglund, J. Agren, and A. Engstrom: J. Phase Equilibria., 2000, vol. 21, p. 269

46. C.A. Apple, R.N. Caron, and G. Krauss: Metall. Trans. A, 1974, vol. 5A, pp. 593-99.

47. T. Swarr and G. Krauss: Metall. Trans. A, 1976, vol. 7A, pp. 41-48.

48. Marder AR: Ph.D. Thesis, Lehigh University, 1968.

49. Y. Xia, M. Enomoto, Z. Yang, Z. Li, and C. Zhang: Phil. Mag., 2013, vol. 93 (9), pp. 1095-109.

50. Z.D. Li, G. Miyamoto, Z.G. Yang, and T. Furuhara: Metall. Mat. Trans. A, 2011, vol. 42A, pp. 1586-96.

51. J. Wang, P.J. Van der Wolk, and S. Van der Zwaag: Mater. Trans., 2000, vol. 41 (7), pp. 761-68.

Publisher's Note Springer Nature remains neutral with regard to jurisdictional claims in published maps and institutional affiliations. 